\title{
Exploration of undergraduate preservice teachers' experiences learning advocacy: A mixed-methods study
}

\begin{abstract}
Kelley Massengale ${ }^{1}$, Cherese Childers-McKee ${ }^{2}$ and Aerin Benavides ${ }^{3}$
Abstract: Applying transformational critical advocacy research in college instruction can be a powerful way to engage students in challenging inequity in society and promoting positive changes. Few studies systematically measure the impact of such pedagogy on the development of college students' beliefs about advocacy. In this mixed methods study, we worked with 21 preservice teachers through advocacy letter writing activities and collected data from pre/post surveys and focus group discussions to explore the impact of such pedagogy. The findings indicated that advocacy letter writing was a meaningful activity for preservice teachers, allowing them a professional opportunity to voice their concerns about personally meaningful issues to entities in power. A significant correlation was found between baseline advocacy experiences and baseline advocacy beliefs, suggesting that the teaching of advocacy, when combined with opportunities for meaningful practice, can contribute to shifts in belief about the importance of advocating.
\end{abstract}

Keywords: transformative education, teacher education, advocacy, mixed methods research

\section{Introduction}

Educators recognize the importance of equipping students with a variety of skills that will benefit them in their personal and professional lives. The ability to advocate for self and others represents a useful strategy for fostering personal empowerment and effecting social change. Scholars in a variety of fields have integrated advocacy-type activities in coursework. Some of the more prominent have been in the health profession (Hearne, 2008; Radius, Galer-Unti, \& Tappe, 2009) and in teacher education (Athanases \& De Oliveira, 2008, Grymes, 2007). In teaching students about advocacy and providing opportunities for them to engage in advocacy within class activities, an underlying assumption is that as students learn about advocacy, their belief in the benefit and worth of advocating will naturally follow. Yet, there have been few studies that systematically measure shifts in beliefs as undergraduate students actively engage in advocacy activities. Our study design was influenced by the overarching questions of how individuals might take up a critical advocacy lens and how undergraduate students might be empowered to advocate in their personal and professional lives. In this study, we described how advocacy activities were integrated in a college course and examined the impact of such activities on the development of undergraduate preservice teachers' advocacy beliefs.

\footnotetext{
${ }^{1}$ Doctoral Student, University of North Carolina at Greensboro, Department of Public Health Education, kemassen@uncg.edu

${ }^{2}$ Doctoral Student, University of North Carolina at Greensboro, Department of Educational Leadership and Cultural

Foundations,cdchilde@uncg.edu

${ }^{3}$ Doctoral Student, University of North Carolina at Greensboro, Department of Teacher Education and Higher Education, awbenavi@uncg.edu
} 


\section{Review of Literature}

\section{Theoretical framework}

The philosophy that undergirds our inquiry into the topic of undergraduate students' experiences learning advocacy is informed by critical advocacy research (Shields, 2012). Through this particular lens, we foreground the role of critical epistemologies in addressing "questions of inequity and disparity" for the purpose of advocating for "policies and practices that can lead to economic, ecological, and human justice, and a sustainable global future" (Shields, 2012, p. 3). We define advocacy in line with Shields as "taking a stance on behalf of a person or a position in which one believes" ( $p$ 6). Critical advocacy research reflects a commitment to social justice, empowerment, and change, while upholding standards for conducting research that are rigorous, contextual, and meaningful.

This study is situated within a transformative mixed methods paradigm in which the researchers acknowledge the workings of power in all parts of the research process (Mertens, 2003, 2007). This particular design is appropriate for a study of teaching advocacy to undergraduate students in that we seek to "conduct research that is change-oriented and seeks to advance social justice causes by identifying power imbalances and empowering individuals and/or communities" (Creswell \& Plano Clark, 2011, p. 96). Therefore, research decisions, from research questions to data analysis, reflect a philosophical commitment to empower participants to advocate for themselves and others. This study reflects participatory elements of a transformative design (Mertens, 2003, 2007, 2010, 2012). Also, it represents an important step toward empowering undergraduate students to cultivate the critical consciousness necessary to engage in transformative-emancipatory work in their personal and professional lives. An exploration of the processes by which individuals take up a critical advocacy lens and come to think of themselves as advocates holds implications for educators of undergraduate students in a variety of fields including education, public health, social work, and many more.

\section{Advocacy in teacher education}

Both critical advocacy teaching and the transformative mixed methods design mirror conceptualizations of advocacy in teacher education. In a review of standards used by several teacher education programs, Grymes (2007) found that typical standards reflected a broad range of activities that could be considered advocacy such as, policy-related advocacy, advocacy based on developing relationships and trust with families, and advocating for individual children with special needs. She concluded that although commonly used standards already reflect the need for advocacy, teacher education programs must continue to equip teachers with necessary skills to be advocates for themselves, their students, and their school communities. Sleeter (2008) draws similar parallels by including teacher activism and advocacy as one of two broad strands critical in educating teachers for equity and diversity

In order to explore teachers' perceptions of advocacy, Althanases and de Oliveira (2008) conducted focus groups of a representative group of new teachers to find out how and why teachers engaged in classroom advocacy. Advocacy, as defined by participants and researchers, included meeting diverse needs of students, hunting for resources, tutoring, increased parent communication, fieldtrips, and creating a culture club. Teachers identified connections between their acts of advocacy and the training acquired in their teacher education programs. Athanases 
and de Oliveira (2008) conclude that an advocate views "all aspects of school as problematic rather than given," "learn[s] to locate expertise inside oneself," and can envision how schools can more effectively meet all students' needs (p. 68). Similarly, Silverman (2010) draws connections between preservice teachers' beliefs about diversity and feelings of efficacy in advocating for change. In her review of the literature, she contends teacher education programs should increase their focus on helping teachers' develop feelings of responsibility and commitment to advocate for diversity. Data found that many survey respondents, though they identified themselves as advocates, felt low personal responsibility to advocate. Additional research is needed to explore the nuances of how the responsibility and desire to advocate are cultivated within undergraduate courses.

\section{Teaching advocacy}

The teaching of advocacy in undergraduate classrooms has been used for multiple purposes. As a pedagogical strategy, advocacy-related instruction is thought to increase student engagement and motivation in the classroom (Beacham \& Shambaugh, 2007). Beacham and Shambaugh (2007) describe two case studies in which advocacy activities represented a type of PBL (problem-based learning) in both an education graduate level course and an interior design undergraduate level course. They describe that advocacy-related activities fostered increased student engagement through enhancing "relevancy, challenge, and uniqueness" (p. 316) of instruction. Beacham and Shambaugh conclude that advocacy as both a teaching strategy and a learning objective enhanced students' feelings of agency and empowerment.

As service learning becomes increasingly common in undergraduate courses (Brundiers, Wiek, Redman, 2010; Butin, 2006; Cawthorn, Leege, \& Congdon, 2011), the teaching of advocacy becomes a critical component in preparing students for community engagement. Preservice teachers have difficulty making connections between personal experiences of social service and larger societal issues, and this can be made explicit as a course component based on advocacy (Wade, 2003). Bridging theories on advocacy and service learning, Berke, BoydSoisson, Voorhees, \& Reininga (2010) describe the experiences of undergraduate family science students who were matched with community agencies and worked on collaborative advocacy projects throughout the semester. Students experienced positive benefits from engaging in advocacy activities including knowledge of working with agencies and an increased understanding of advocacy as a macro-level process. The existing studies on the teaching of advocacy in undergraduate classrooms emphasize the benefits of advocacy. Further study is needed to better describe variations in effectiveness for different forms of advocacy, variations across content areas, and the role of advocacy experiences in shifting beliefs about the benefits of advocating. Additionally, studies on the teaching of advocacy in undergraduate classrooms would benefit from a more robust theory of the stages of advocacy development for undergraduate students. This study provides both an analysis of the effectiveness of an advocacy lesson and activity in shifting beliefs about advocacy and offers a model of the stages of development of beliefs in advocacy.

\section{Advocacy letter writing}

Advocacy letter writing has been employed to express concerns to people in power and suggest changes on behalf of individuals, minority groups, majority groups, and the environment. 
Syndicated cartoonists who were the recipients of advocacy letters changed their behaviors and began drawing seat belts on their characters when depicting them in vehicles (Mathews \& Dix, 1992). Writing advocacy letters has been suggested as a method for contacting politicians on behalf of legislative health issues (Huntington, 2001), as an effective tool for individuals living with disabilities (White, Thomson, \& Nary, 1997), and as a transformative activity for preservice English for Academic Purposes and English as a Second Language teachers (Morgan, 2009).

Within the undergraduate classroom, advocacy letter writing is a tool for engaging students in social discourse. Advocacy letter writing on the topic of oil and politics has been used as an assignment to integrate political science and English composition skills for first year undergraduate students (Huerta \& Sperry, 2013). Undergraduate nursing students participated in advocacy letter writing as a way to learn about population groups affected by community health topics (Eide, Hahn, Bayne, Allen, \& Swain, 2006). What is unknown about previous undergraduate advocacy assignments is how students' beliefs about advocacy may have been impacted by participating in the assignments. This paper builds upon the existing literature about advocacy letters by describing the experiences and change in advocacy beliefs of a group of undergraduate preservice teachers who were assigned to write advocacy letters to people in power about issues of personal meaning to them.

\section{Course context}

\section{Selected courses}

Preservice teachers who are undergraduate students dually enrolled in a course on both elementary education in seminar/internship and advanced science methodology participated in the study. These particular courses were selected because one is an experiential learning course following a theme of cultural awareness. The other course includes a lesson on how to differentiate between environmental education and environmental advocacy. Students enrolled in these classes may benefit from learning about advocacy as they are preparing to enter the profession of elementary education in which they may need to advocate for individual students, groups of students, or themselves as teachers.

\section{Advocacy lesson}

All students in the courses participated in a lesson on advocacy. The primary instructor of the lesson, a doctoral student with experience teaching undergraduate public health courses, had previously delivered similar lessons to students enrolled in public health courses. Three other instructors from the Teacher Education and Educational Leadership \& Cultural Foundations departments joined the lesson discussions to share their own perspectives and experiences.

The interactive lecture began by asking students to share, with a classmate, a memory from a time when they or someone that they cared about did not have something that was needed or was treated unfairly. Students were asked to reflect on how they felt at the time, whether or not any action was taken, whether it was difficult to take action, feelings about the outcome of the situation, and whether they would act differently in a similar situation in the future. Next, the primary instructor shared an example from her own experience then invited students to share their experiences with the entire class if they wanted. Students shared a wide range of experiences and some students' stories revealed details about their lives and personal values that 
were previously unknown to their peers and instructors. The class engaged in a discussion on how these experiences were acts of advocacy or revealed a time when advocacy could have potentially changed the outcome.

The lesson then proceeded by including the preservice teachers' input in defining advocacy, determining what an advocate does, and in brainstorming reasons to advocate. Preservice teachers learned about the phases of advocacy (defining and researching an issue, planning advocacy activities, thinking of all possible solutions and outcomes, advocating, evaluating the act of advocacy, then planning for any future actions) and role-played effective and ineffective advocacy. The instructor led a discussion on what might happen if the act of advocacy had unintended consequences or made the situation worse. Preservice teachers discussed how they might act as advocates in their future roles as in-service teachers and brainstormed when presented with the scenario of how they could advocate for elementary school student fieldtrips given district budget restraints.

Next, in small groups, preservice teachers evaluated a case study about community development near a marsh and demonstrated critical thinking in determining how different acts of advocacy might lead to different outcomes and what any unintended consequences of advocacy might be. Preservice teachers listed pros and cons to appealing a decision made by local legislatures, involving an outside advocacy organization, and taking no action. Preservice teachers also listed alternate actions and acts of advocacy they identified on their own. Through discussion of the pros and cons they brainstormed, material taught earlier in the lesson about characteristics of effective and ineffective advocates was emphasized. Throughout the entire lesson, preservice teachers were encouraged to ask questions and to share any of their own experiences with advocacy. The lesson concluded with a group discussion of a news story about a Polish art student who advocated for his own art by hanging it in a museum (Scislowska, 2012). The news story allowed the students to review what they had learned during the lesson and to connect it to the real life experience of a student, someone they may have been able to relate to.

\section{Advocacy letter writing activity}

At the end of the advocacy lesson, preservice teachers were given an assignment to write advocacy letters of their own. Example letters and suggested formats were shared. The phases of advocacy discussed during the lecture were reviewed in dissecting example letters. Participants were instructed to each choose an issue of personal meaning to research and compose an advocacy letter requesting a change in the status quo. No restrictions were placed on the advocacy topics; participants were encouraged to reflect on what was important to them in their personal lives noting that the issue could be something that affects a small group of people, a large population, a specific community such as a student's hometown, or one that was relevant for policy makers. Preservice teachers were asked to reflect on what could be done to improve the issues of choice and to determine who had the power to make the changes happen. To increase the likelihood of receiving responses to their letters, participants were encouraged to send their letters to local entities with the power to enact changes on their chosen topics. They were given one week to research their issues, write, and send their letters. Proof of letter submission was required to receive credit for having completed the course assignment. 


\section{Methods}

\section{Participants}

The preservice teachers participating in the study were enrolled as undergraduate students at a medium-sized university in a Southeastern state. All of the students were seniors, $(n=22$, average age $=22$ years old) pursuing Elementary Education as a major and earning a state Environmental Educator Certification. The participants were all female and self-identified as: 90\% Christian, 81\% White, 43\% raised in a rural environment, and 57\% raised in a suburban environment. Average self-reported annual income of the participants' families of origin was measured using income categories from the US Census with 52\% reporting income in the upper two-fifths, $29 \%$ in the mid fifth, and $19 \%$ in the lower four fifths.

\section{Study design}

In acknowledging the multifaceted nature of the concept of advocacy, a mixed methods transformative approach could best capture the complexity of the issue (Mertens, 2012) and reflect the purpose of empowering and effecting change in participants' understanding of advocacy. The research design included qualitative research and quantitative research in parallel strands (see Figure 1). Per Creswell and Plano Clark (2011), the start of many mixed methods studies falls between fixed and emergent designs. Our study also fell on this continuum with both fixed and emergent characteristics. As an emergent mixed methods design (Creswell \& Plano Clark, 2011), the guiding original transformative study design was altered to include a quantitative/qualitative post survey; this was added during the research process. We as researchers felt the data obtained in the pre-intervention survey about advocacy beliefs would be important to collect again after the focus group discussions, in order to specifically address our research question about significant difference in advocacy beliefs. The one eight-item question on the post-intervention survey was taken from the pre-intervention survey instrument. We decided to add another qualitative question at the end of our post-intervention survey as themes of growth in advocacy emerged from the focus groups. We wanted to give participants a chance to provide any final words on their experiences.

The following research questions were constructed to represent a blend of both quantitative and qualitative strands and guided the investigation of students' experiences learning advocacy: 1) Is there a statistically significant change in preservice teachers' beliefs about engaging in advocacy through a lesson on advocacy and a letter writing activity as evidenced by responses on a pre- and post-intervention survey? 2) In what ways do the focus groups' results explain any differences between pre- and post-intervention survey responses? 3) What meanings do preservice teachers make of advocacy and the advocacy letter writing activity? 


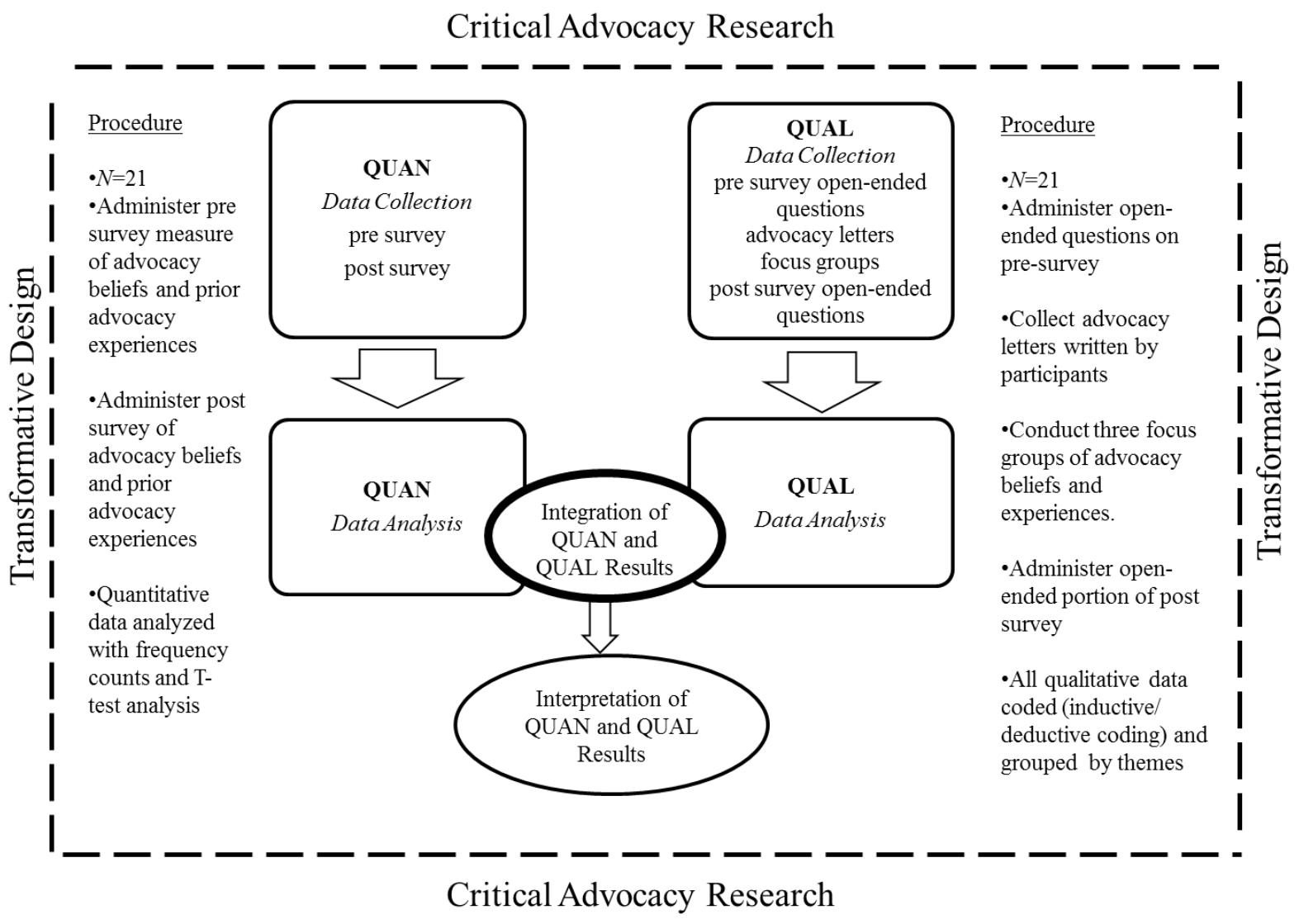

Figure 1. Research Design

\section{Data collection}

Research participants took a written pre-intervention survey to express their prior participation in advocacy and express their beliefs about advocacy. Next, participants engaged in a lesson on advocacy conducted by the research team, then were asked to write a letter to a local entity advocating for change on a topic of personal meaning to the participant. Three weeks later, participants were asked to join a focus group to have an opportunity to further discuss the ways in which they understood their experiences participating in the lesson and writing the advocacy letter. Finally, all participants took a written post-intervention survey.

\section{Pre- and post-intervention survey}

Part One of the participant survey contained questions modeled after those on the Social Issues Advocacy Scale [SIAS] (Nilsson, Marszalek, Linnemeyer, Bahner, \& Misialek, 2011). The SIAS, created to assess behaviors and attitudes about advocating on social justice issues, measured activities and beliefs relevant for most any field or discipline (Nilsson et al., 2011). The instrument used a Likert-type scale to measure participation in various advocacy-related activities or agreement with specific beliefs and demonstrated good internal validity (Nilsson et al., 2011). Modifications were made to collect similar data without a Likert-type scale. Following these quantitative questions on baseline advocacy activities and beliefs, two open- 
ended qualitative questions allowed the participants to describe prior undergraduate learning experiences and course assignments in which they may have learned about advocacy.

Part Two of the participant survey contained questions modified from those on the Social Justice Advocacy Scale which was designed to measure counselors' social justice advocacy abilities (Dean, 2009). Modifications were made to measure beliefs about aspects of social justice advocacy relevant to preservice teachers. The responses to the 25 Likert-type scale questions in this portion of the survey were tested for internal reliability, and for these items it was high, with a Chronbach's Alpha of .895. The exact cutoff for what is considered high reliability is debated, but it is generally accepted that high reliability in Chronbach's Alpha is anything above .8 (Field, 2013).

\section{Focus groups}

All participants in the study submitted their letters to an instructor by hard copy or email. The research team met the day before focus group discussions to read and sort the letters into three groups of mixed topics. The letters were written to a range of addressees, from local officials to large corporations, and were evenly distributed among the three focus groups, purposefully giving each focus group a variety of topics.

The items included in the focus group protocol were guided by the qualitative research question, What meanings do preservice teachers make of advocacy and the advocacy letter writing activity? Consistent with the convergent parallel research design, the focus group protocol was written at the onset of the study. During the three focus groups, students first introduced themselves and the topic of their letters. The rest of the focus group was guided by a semi-structured interview protocol.

In analyzing focus group transcripts, initial coding reflected Hatch's (2002) conceptualization of an inductive analysis approach that identifies patterns of meaning in data in order to make statements about the phenomenon under investigation. Interrater reliability was assured by all three researchers listening to audio recordings of all three focus group sessions before coding data, then reaching consensus on coding within each transcript. After initial open coding, each transcript was read multiple times and recoded by each member of the research team to identify additional codes in efforts to triangulate interpretations. Then, all codes were placed into categories which were then thematized, paying close attention to outliers that ran counter to initial interpretations (Hatch, 2002; Strauss \& Corbin, 1998)

\section{Results}

\section{Changes in advocacy beliefs}

Through analysis of quantitative data collected on the pre- and post-intervention surveys during the study, we sought to address our first research question to determine if there was a statistically significant change in preservice teachers' beliefs about engaging in advocacy through a lesson on advocacy and a letter writing activity. Prior to participation in the advocacy lesson and letter writing activity, participants reported 96 total acts of advocacy from among 14 different advocacy activities. Participants, on average, had previously engaged in 4 to 5 different types of advocacy activities with discussing bills and legislative issues of personal importance with family and friends $(81 \%)$, voting in local elections $(62 \%)$, and using social media to influence 
others regarding issues they cared about $(52 \%)$ being the most popular activities. Advocacy experience was found to have a positive correlation $(p=.011)$ with average scores on the Likerttype scale questions about advocacy beliefs.

Prior to the advocacy lesson, $76 \%$ of students had previously learned about advocacy in another undergraduate course. Several students named a required Educational Leadership and Cultural Foundations course which explored education as a social institution and advocating for racially, culturally, and linguistically diverse groups. One preservice teacher described a course activity that required students to attend an event or setting outside their comfort zones as one which taught her about advocacy. Others mentioned learning about environmental advocacy or Response to Intervention (RTI) to accommodate individual learning needs.

The preservice teachers, on average, agreed with 5 out of the 8 advocacy belief items in Part One of the survey prior to participation in the letter writing activity. Almost one third (29\%) agreed with all eight items. The first four items were beliefs related to the effects of societal forces on health, wellbeing, and educational performance as well as the effects of state and federal policy on access to education and social services; the majority of participants $(70 \%)$ agreed with each statement. The highest agreement was with the belief in societal forces affecting access to education and resources (95\% agreed). The last four items were beliefs in the professional responsibility to confront those who discriminate against the elderly, disabled, those of a different culture or ethnicity, or of a different sexual orientation.

We also found a significant correlation between individual advocacy experience and beliefs scores (number of items respondents agreed with on Part One of the pre-intervention survey) and the average individual score on the Likert-type scale questions in Part Two. The overall mean frequency score for beliefs and experiences in advocacy for Part One was 4.57. The overall mean Likert score for Part Two on the pre-intervention survey was 2.6, on a scale of 0 11. The range of mean Likert scores for the preservice teachers was from $0.36-5.68$. Testing for a correlation between beliefs and experiences scores in Part One, and beliefs scores in Part Two, a positive correlation is statistically significant $(p=.011)$ at the .05 alpha level (2-tailed). The strong baseline positive correlation of experience in advocacy with belief in advocacy in this sample is a compelling argument for recommending that participants experience advocacy as part of transformative coursework.

On average, none of the eight items on both surveys showed a decrease in advocacy beliefs pre-post, and the last four items showed an increase in advocacy beliefs. In an analysis of mean differences (using SPSS paired samples $t$-test) between individual pre- and postintervention survey responses $(n=19)$, there was a statistically significant mean difference increase in the average number of individuals' advocacy beliefs $(\bar{x}$ pre $=0.64, \bar{x}$ post $=0.76, \alpha=$ $\left..05, d f=19, t_{\text {crit }}=-2.62, p=.018\right)$.

\section{Participants' explanations of belief changes}

Qualitative data collected during the focus groups enabled us to address our second research question in which we sought to determine in what ways the focus groups' results explained any differences between pre- and post-intervention survey responses. In all three focus groups, facilitators began with the same focus group protocol-although we also permitted participants to introduce and address additional topics that came up in the course of conversation. In this way focus groups were semi-structured in order to encourage a more participatory style that in some instances allowed participants to control the flow of the conversation. Due to time constraints 
focus groups lasted approximately an hour and participants were encouraged to discuss their feelings and experiences with the letter writing activity and any plans for future advocacy.

Topics ranged from training the police force in the state to recognize and prevent human trafficking to improving maintenance at a breezeway at the participant's apartment complex. One preservice teacher said she used a "sandwich" of a compliment, the meat of the letter, and then ended with a compliment, to request a large cereal manufacturer omit a potentially cancer causing chemical from their cereal, and soon after her comment, another student in her group used the same terminology, "sandwich," to describe the letter she wrote. Clearly, as focus group discussion progressed, there was listening, reflection, and learning taking place. One student said she knew she should have researched before writing to Parking Services, but her letter was from emotion. The act of discussing the process of writing reminded participants of the recommended steps from the advocacy lesson. There was explicit expression of learning through discussion, as a student exclaimed, "Oh, I hadn't thought of that before. .."

Over 30 individual codes were generated from the focus group transcripts, then grouped thematically into the following 6 categories: 1) Awareness of the benefits of advocacy, 2) Negotiating who benefits from advocacy, 3) Affective dimensions of advocacy, 4) Barriers to advocacy, 5) Negotiating the process of becoming an advocate, and 6) Embracing advocacy. While the pre- and post-intervention surveys were crucial to answering the question of whether or not beliefs about advocacy changed, the focus groups contributed to an understanding of the nuances and idiosyncrasies of changes that occurred in participants' awareness, beliefs, or action around questions of advocacy (see Figure 2).

Awareness of the Benefits of Advocacy. Participants described the extent to which the advocacy activities shifted their beliefs and perspectives about advocacy or increased their awareness of a particular issue. Shifts in awareness occurred in several important areas. Participants expressed that they became more aware of how to advocate and why advocacy is important. One described, "I think this assignment opened up my eyes to the importance of advocacy. Even if your advocacy does not result in change, you put your voice out there and stood up for what you believe in." Another remarked, "It brought to my attention how showing you care can have an overall effect." Therefore, an awareness of ways to effectively advocate as well as the belief that advocacy is important work in tandem in the process of becoming an effective advocate.

Negotiating the Process of Becoming an Advocate. This category represents a broad theme that encompasses data related to specific advocacy skills learned during the advocacy lesson and how students responded to the process of learning to be advocates. Learning about the process of advocacy reflected one of the most important components of their burgeoning advocacy development. One participant stated, "First time advocating for anything. Interesting to learn the process." Another stated, "It's a very authentic learning experience." Participants also added that the experience gave them added knowledge about different ways to advocate and broadened their perspective of what advocacy is. One stated, "When I thought of an advocate, a lot of times I thought of an extremist." Therefore, many participants viewed letter writing as an effective way to advocate that was potentially lower risk than other forms of advocacy like marching or protesting. While most participants expressed that they enjoyed the lesson and felt it was beneficial information to know, it was not assumed that this implied they would necessarily engage in future advocacy. Therefore, additional themes were identified that further contribute to the advocacy development process. 


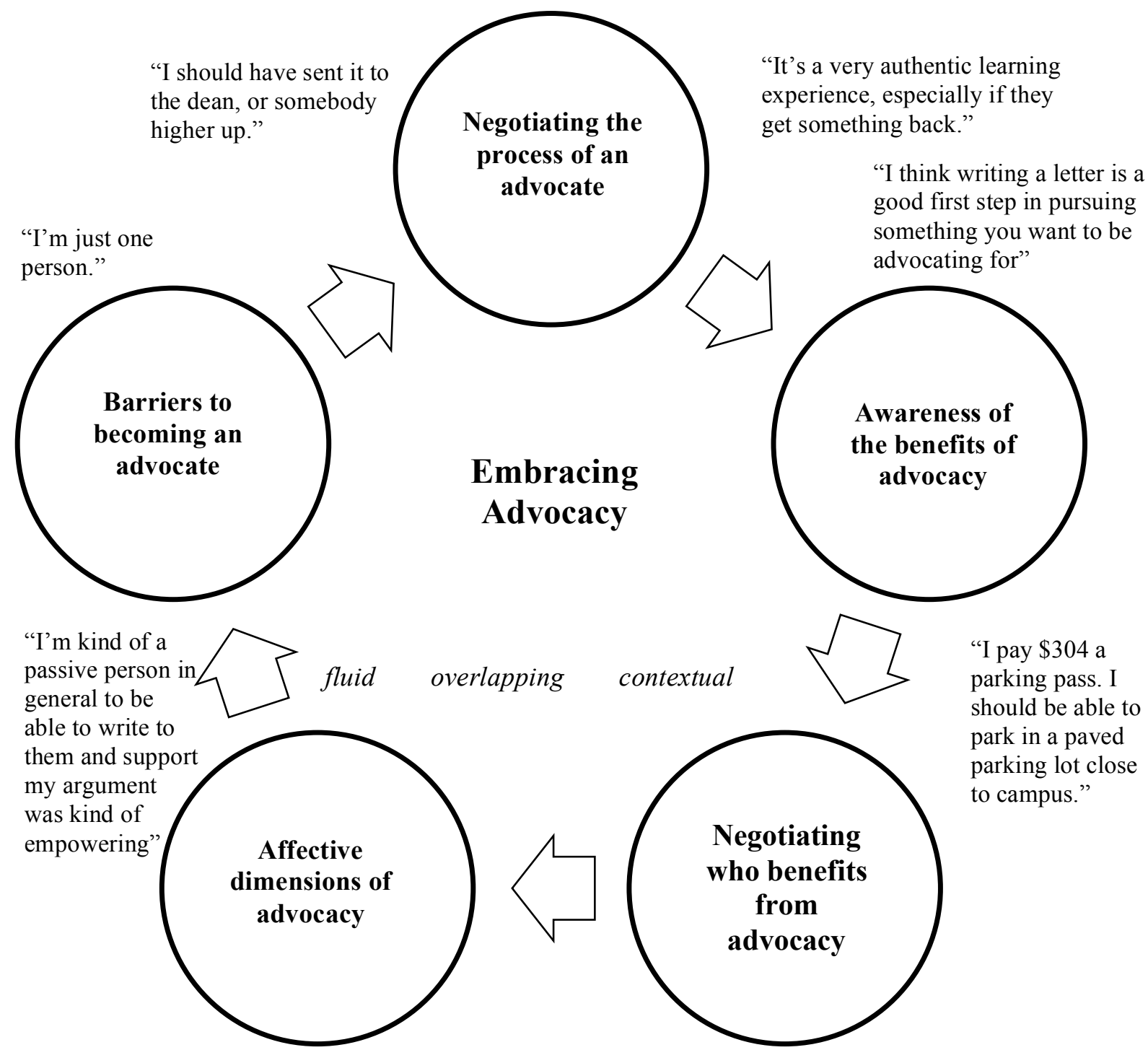

Figure 2. Advocacy Development Model

Negotiating Who Benefits from Advocacy. In keeping with the theoretical framework that privileges participants' involvement in the research process, participants possessed complete control over the topic of their letters. The choice of whether to advocate for self or others represented a salient theme throughout all three focus groups. One participant stated, "The activity was very beneficial and helped me learn the importance of advocating for myself and what's important to me." Many other participants echoed similar sentiments, while others chose to advocate for issues that would impact others more than themselves. While it could be interpreted that negotiating for self shows a more novice understanding of advocacy, upon deeper analysis of focus group responses, we suggest that negotiating the beneficiary of advocacy activity represents a key component in the development of an advocacy identity. In learning to advocate for themselves, some participants developed a greater confidence in advocating for others in future endeavors.

Affective Dimensions of Advocacy. The feelings and emotions associated with engaging in advocacy represented recurrent themes in all focus groups. Participants expressed the personal 
satisfaction gained through advocating, feelings of frustration or disappointment at not receiving a response to a letter, and the sentiment that being able to advocate for something personally meaningful gave them a voice. Affective dimensions of advocacy were also critically important in fostering feelings of empowerment among participants. One student shared, "I'm kind of a passive person in general...so to be able to write to them and support my argument was kind of empowering." One stated, "It's been a continual frustration. This letter was a great way to get if off my chest and I felt better when I was done writing it, cuz I was able to vent." Another remarked, "When I sent this letter and received feedback it made me feel like I had taken a step towards change and that felt good." As illustrated in this response, responses to letters seemed to play an integral part in participants' feelings about the experience. Those who received responses expressed excitement and feelings of encouragement, while some of those who did not expressed frustration and feelings that letter writing was ineffective. Others who did not receive responses simply expressed a renewed commitment to continue trying. Overall focus group data about the affective dimension of advocacy suggest that students who expressed strong emotions about a particular topic were more empowered to express their concerns and take action for change.

Barriers to Advocacy. Although barriers to advocacy were mentioned at least once in each focus group, participants' hesitancy in taking up identities as advocates was more pronounced in one focus group discussion in particular. Although negative sentiments about advocacy were primarily espoused by two individuals in the group, others silently concurred with certain statements. One participant stated, "You put all this time and hope into researching for a letter and the other end doesn't even care to say anything back." Another responded, "The majority of the time they're gonna do whatever they wanna do and it has to do with money." Several participants worried that their one letter would not be significant enough to effect change. We suggest that in developing an understanding of the process by which participants develop an advocacy identity, negotiating barriers to advocacy represents a real and valid concern.

\section{Meaningfulness of advocacy letters}

To address our final research question, "what meanings do preservice teachers make of advocacy and the advocacy letter writing activity," we considered our analysis of the advocacy letters themselves, the quantitative and qualitative analysis previously described, and a qualitative question added to the post-intervention survey. As part of the study intervention, students were asked to compose and send to an entity in power a letter on a personally meaningful topic about which they wanted to advocate. The participants $(N=22)$ who wrote advocacy letters all provided evidence that their letters had been written and sent. Some provided the letter to researchers to mail, while others produced a screen shot or confirmation page of the letter being sent electronically. Letters were analyzed by topic, beneficiary, and whether or not a response was received. In analyzing the beneficiaries of the letters, or the individual(s) for whom the participant intended to advocate, three categories emerged: self advocacy, advocacy for self and others, and advocacy for others. Letters written to advocate for self included letters written to the university parking services $(n=4)$, letters written to campus dining services $(n=2)$, participant's employer $(n=1)$, and the health insurance provider of a participant $(n=1)$. Letters written for the purpose of self-advocacy represented $37 \%$ of letters written. Interestingly, an equal percentage of letters were written to advocate for others $(37 \%)$. In contrast to self-advocacy letters in which 6 of the 8 letters written related to issues specific to participants' university 
experience, the letters written to advocate for others were more varied. Topics included letters to the state governor $(n=2)$ about increasing funds to state school systems, a letter to a representative about increasing awareness of human trafficking in the state, a letter to a local school superintendent to reduce class size by hiring an additional teacher, a letter to emergency services to provide an ambulance for rural parts of a local county, a letter to a national corporation about the chemical BHT in cereal marketed to children, a letter to a restaurant about working collaboratively with local high schools to employ teens, and a letter to campus disability services to improve ramps and make campus more wheelchair accessible. The remaining (27\%) were written for self and others and represented similar topics to those just described.

Due to the potential influence of rate of response on feelings about advocacy, the letter analysis documented whether or not each participant received a response. Of the 22 letters written, $37 \%(n=8)$ of participants showed proof that they received a response to their letter. All but one of the letters that received a response were written to local entities. The rate of response varied, with some participants receiving immediate responses while others received responses after a couple of weeks. All of the responses were polite and encouraging, some articulated future plans to address the requested change, and others suggested alternatives that the letter writer could explore, but none of the letters promised immediate changes. Although the advocacy letters were a significant piece of the data analysis, there were many questions left unanswered. How did participants choose a topic? How did the classroom lesson and discussion contribute to their letter writing experiences? Did participants perceive letter writing as an act of advocacy? How did receiving a response potentially influence participants' feelings about the experience? Therefore, the focus groups represented an important vehicle for both understanding participants' feelings about the process of letter writing to advocate for a cause, as well as continuing the discussion about advocacy and empowerment.

Two-thirds of all participants responded to the optional open-ended question at the end of the post-intervention survey where participants could have the final say on the project. All responded positively and similarly to tell us just how meaningful the experience was:

"I feel that this assignment was beneficial in educating us about advocacy, and it gave us the opportunity to be an advocate for something we believe in. Because of this project I feel that I am more clear on what advocacy is and different ways you can choose to advocate."

Another student wrote:

"This has helped inform me of advocacy and I now want to advocate more about things I am concerned and passionate about!"

Participants' understandings of and feelings about advocacy fell along a fluid continuum. Although participants reported prior advocacy experiences at baseline, after taking part in the letter writing experience all embraced advocacy to some extent, even those with the lowest baseline scores. This was revealed in a systematic analysis of a cross section of all data collected for each individual. Through both focus groups and post-intervention survey open ended responses, participants identified themselves as advocates, making future plans to advocate, recognizing the complex dimensions of advocacy, expressing they had learned how to advocate, and showing an understanding of the role of power in advocacy. 


\section{Discussion}

\section{Interpreting preservice teachers' prior advocacy experiences}

Preservice teachers' shared experiences prior to participating in the intervention could account for some but not all of the responses on the pre-intervention survey. Each of the participants had experience as an intern in local public schools for over three semesters prior to completing the survey at baseline. The overwhelming agreement with the statement that society affects access to education and resources was not surprising as teaching was their specialty, and they had firsthand knowledge of inequities in local area public schools.

Due to their common major and required coursework, participants typically take the same core group of classes. Despite having been enrolled in the same classes throughout the past year, participants had vague and inconsistent understandings of advocacy prior to participating in advocacy activities in this study. Activities in prior courses that some participants recognized as advocacy activities were not recalled by others. This finding supports the justification for including specific instruction on advocacy in undergraduate courses coupled with the opportunity to advocate for something.

Results from all phases of this study provide encouragement for continuing to incorporate advocacy lessons and assignments in undergraduate courses. First, results suggest that the process of advocating for something personally meaningful empowered participants and transformed their ideas about advocacy. Therefore, the combination of the advocacy lesson, actual practice advocating for something personally important, and the experience of discussing the process with others contributed to participants' greater understanding of the process and importance of advocacy. Second, beliefs shifted through learning about advocacy and engaging in advocacy experiences. The cross analysis of quantitative and qualitative data shows the transformation that occurred in students' beliefs and understandings of advocacy. These findings corroborate and expand upon other research of students' learning of advocacy (Beacham \& Shambaugh, 2007; Berke et al., 2010) by explicitly drawing connections between experiences engaging in advocacy and beliefs about advocacy. Further, the findings illustrate the usefulness of advocacy teaching as a pedagogical intervention to connect theory to practice or illustrate the real-world usefulness of advocacy in professional settings. As some students indicated that writing and sending the advocacy letters was the first time they had ever advocated for something, the opportunity to advocate for something within the context of an undergraduate course allowed them the guidance and experience of their instructors as they interpreted their own actions and reflected on the experience of advocating for the first time. Having had the opportunity to advocate in the context of their own learning experiences, the preservice teachers may feel more confident and prepared to advocate in a professional setting once they are no longer in the student role.

Finally, learning to advocate is a fluid, contextual process in which discussion, reflection, and the co-construction of ideas is important. Integrating data from all results, we have theorized a model for understanding advocacy development or the way in which individuals come to view themselves as advocates. Within this model (Figure 2), individuals develop an awareness of the benefits of advocacy while negotiating various components within the process of becoming an advocate including who benefits (self, other, or both), affective feelings about advocacy, and barriers and obstacles to seeing oneself as an advocate, before finally embracing an identity as an advocate. Although the model is circular, it should not be interpreted as an illustration of 
sequential steps that individuals go through on the path to becoming an advocate; instead, embracing a critical advocacy lens involves a fluid, contextual, and interconnected process of negotiation influenced by many components.

\section{Limitations of the study}

While the participants found the advocacy lesson and letter writing activity to have personal meaning for them, the sample size was small and relatively homogenous. More research is needed to understand if the same lesson and activity would be an effective way to teach a more diverse group of undergraduate students about advocacy. The timeframe of the study required all activities to be complete within one semester. This short timeline did not allow for providing participants with much guidance on continuing to advocate for their specific topics after their initial letters were sent and any initial replies were received. Also, detailed discussions of power, privilege, social justice, and inequality require more time than we were afforded in the research site. While these components of advocacy represented an integral part of our research lens, due to time restrictions, they were not explicitly presented to participants during the advocacy lesson. Therefore, future research in this area could explore the ways in which discussions of social justice influence the choices of letter topics and the overall development of advocacy in undergraduate students. Between the pre- and post-intervention surveys, the students read and discussed a book on workplace diversity for a separate assignment. Their experiences reading the book may have also contributed to the increased number of students who indicated on the postintervention survey that they would feel compelled to act if a colleague discriminated against others based on differences in culture or ethnicity.

\section{Recommendations for teaching advocacy}

Incorporating lessons and class discussions about advocacy in undergraduate courses is encouraged as a way for students to gain awareness on when and how they may advocate for themselves and others in situations when they do and do not have power over others. Lessons on advocacy may be most effective when they involve both small and large group discussions so that students who are not comfortable sharing personal advocacy experiences with the larger group may still have the opportunity to share with others. Examples of effective and ineffective advocacy from students' intended careers offers suggestions to students on ways they themselves might act as advocates. A case study example may provide students with the opportunity to engage in critical thinking about the potential outcomes and consequences of different acts of advocacy that could all occur in response to the same situation reinforcing advocacy concepts previously learned during a lesson. Letter writing assignments have the potential to teach students about the importance of research and professionalism when contacting entities in power to make changes and allow students the chance to practice advocacy concepts examined during a class lesson. Allowing students to choose the issues for which they advocate naturally encourages students to become personally invested in the outcomes. Visiting the topic of advocacy over multiple course meetings allows students to see how advocacy is applied in multiple contexts and provides the students with guidance as they interpret their own advocacy experiences and develop their own identities as advocates. While this study enrolled students in the field of education, undergraduate students in any discipline may benefit from learning about advocacy as it relates both to their fields of study and to their personal lives. 
Instructors who incorporate advocacy lessons and activities in their undergraduate courses may benefit from preparing for a range of emotions and reactions students may experience when assigned to contact entities in power to encourage a change in the status qua. The portion of students in our study who contacted campus service providers for parking, dining, and accessibility services demonstrates that students are continually reflecting on and interpreting their college experiences of which instructors play integral roles. During focus groups, some students expressed disillusionment with local community leaders in power while others expressed discouraging thoughts about the potential change that could come from their seemingly meager acts of advocacy. Historical and current examples of successful advocates can remind students of their own potential as advocates. Still, students demonstrated creativity and thoughtfulness in their words and advocacy actions, leaving us encouraged as they will soon grow in their advocacy identities as teachers.

\section{Conclusion}

In their future roles as in-service teachers, preservice teachers will encounter many instances when advocating for a change in the status quo may benefit an individual student, an entire class, a school, or school system, or themselves as teachers. Some of the instances may require immediate action, others may require cycles of research, planning, action, and reflection to achieve the greatest possible impact. Empowering preservice teachers with effective advocacy skills before they become in-service teachers may give them the confidence to advocate in both their professional careers and in their personal lives. Specific advocacy instruction along with an advocacy letter writing assignment is an effective combination for introducing undergraduate students to the topic of advocacy, providing guidance as students advocate, possibly for the first time, and grow into their identities as advocates.

\section{Acknowledgments}

We acknowledge our preservice teachers for their participation and for providing us with the privilege of understanding their advocacy experiences, some of which were deeply personal. We appreciate the support for our study of Dr. Melony Allen and Dr. Catherine Matthews. We are grateful for the support and guidance of Dr. Ye He.

\section{References}

Athanases, S., \& De Oliveira, L. (2008). Advocacy for equity in classrooms and beyond: New teachers' challenges and responses. The Teachers College Record, 110(1), 64-104.

Beacham, C. V., \& Shambaugh, N. (2007). Advocacy as a problem-based learning (PBL) teaching strategy. International Journal of Teaching and Learning in Higher Education, 19(3), 315-324.

Berke, D.L., Boyd-Soisson, E. F., Voorhees, A. N., \& Reininga, E. W. (2010). Advocacy as Service-Learning. Family Science Review, 15(1), 13-30. 
Massengale, K., Childers-McKee, C., \& Benavides, A.

Brundiers, K., Wiek, A., \& Redman, C. L. (2010). Real-world learning opportunities in sustainability: from classroom into the real world. International Journal of Sustainability in Higher Education, 11(4), 308-324. doi: 10.1108/14676371011077540

Butin, D. W. (2006). The limits of service-learning in higher education. The Review of Higher Education, 29(4), 473-498. doi: 10.1353/rhe.2006.0025

Cawthorn, M., Leege, L., \& Congdon, E. (2011). Improving learning outcomes in large environmental science classrooms through short-term service-learning projects. Journal of Environmental Studies and Sciences, 1(1), 75-87. doi: 10.1007/s13412-011-0001-8

Creswell, J. W. \& Plano Clark, V. L. (2011). Designing and conducting mixed methods research (2nd ed.). Thousand Oaks, CA: Sage.

Dean, J. K. (2009). Quantifying social justice advocacy competency: Development of the social justice advocacy scale. (Unpublished PhD). Georgia State University, Atlanta. Retrieved from http://scholarworks.gsu.edu/cps_diss/40/

Eide, P. J., Hahn, L., Bayne, T., Allen, C. B., \& Swain, D. (2006). The population-focused analysis project for teaching community health. Nursing Education Perspectives, 27(1), 22-27.

Field, A. (2013) Discovering statistics using IBM SPSS (4th ed.). London: SAGE

Grymes, J. M. (2007). Advocacy and teacher preparation programs. Childhood Education, 84(2), 94-D.

Hatch, J. (2002). Doing qualitative research in education settings. Albany, NY: State University of New York Press.

Hearne, S. A. (2008). Practice-based teaching for health policy action and advocacy. Public Health Reports, 123(Suppl 2), 65.

Huerta, J. C., \& Sperry, R. (2013). Pulling it together: Using integrative assignments as empirical direct measures of student learning for learning community program assessment. Learning Communities Research and Practice, 1(1), 16.

Huntington, C. G. (2001). Legislative advocacy for health professions educators. Education for Health Change in Learning \& Practice, 14(2), 241-250. doi: 10.1080/13576280110059228

Mathews, R. M., \& Dix, M. (1992). Behavior change in the funny papers: Feedback to cartoonists on safety belt use. Journal of Applied Behavior Analysis, 25(4), 769-775. doi: 10.1901/jaba.1992.25-769

Mertens, D. (2003). Mixed methods and the politics of human research: The transformativeemancipatory perspective. In A. Tashakkori \& C. Teddlie (Eds.), Handbook of mixed methods in social and behavioral research (pp. 135-164). Thousand Oaks, CA: Sage. 
Massengale, K., Childers-McKee, C., \& Benavides, A.

Mertens, D. (2007). Transformative paradigm: Mixed methods and social justice. Journal of Mixed Methods Research, 1(3), 212-225. doi: 10.1177/1558689807302811

Mertens, D. (2010). Transformative mixed methods research. Qualitative Inquiry, 16(6), 469474. doi: $10.1177 / 1077800410364612$

Mertens, D. M. (2012). What comes first? The paradigm or the approach? Journal of Mixed Methods Research, 6(4), 255-257. doi: 10.1177/1558689812461574

Morgan, B. (2009). Fostering transformative practitioners for critical EAP: Possibilities and challenges. Journal of English for Academic Purposes, 8(2), 86-99.

doi: 10.1016/j.jeap.2008.09.001

Nilsson, J. E., Marszalek, J. M., Linnemeyer, R. M., Bahner, A. D., \& Misialek, L. H. (2011). Development and assessment of the social issues advocacy scale. Educational and Psychological Measurement, 71(1), 258-275. doi: 10.1177/0013164410391581

Radius, S. M., Galer-Unti, R. A., \& Tappe, M. K. (2009). Educating for advocacy: recommendations for professional preparation and development based on a needs and capacity assessment of health education faculty. Health promotion practice, 10(1), 83-91. doi: $10.1177 / 1524839907306407$

Scislowska, M. (2012, January 4). Polish art student hangs own painting in museum. Chicago Sun-Times. Retrieved from http://www.suntimes.com/news/world/9809511-418/polish-artstudent-hangs-own-painting-in-museum.html

Shields, C. (2012). Critical qualitative research. In S. Steinberg \& G. Cannella (Eds.), Critical Qualitative Research Reader (pp. 104-114). New York, NY: Peter Lang.

Silverman, S. K. (2010). What is diversity? An inquiry into preservice teacher beliefs. American Educational Research Journal, 47(2), 292-329. doi: 10.3102/0002831210365096

Sleeter, C. (2008). Equity, democracy, and neoliberal assaults on teacher education. Teaching and Teacher Education, 24(8), 1947-1957. doi: 10.1016/j.tate.2008.04.003

Strauss, A. L., \& Corbin, J. M. (1998). Basics of qualitative research: Techniques and procedures for developing grounded theory. Thousand Oaks: Sage Publications.

Wade, R. (2003). Teaching preservice social studies teachers to be advocates for social change. Social Studies 94(3), 129-133. doi: 10.1080/00377990309600195

White, G. W., Thomson, R. J., \& Nary, D. E. (1997). An empirical analysis of the effects of a self-administered advocacy letter training program. Rehabilitation Counseling Bulletin, 41(2), 74-87. 